\title{
Introducing 'Re-coupling': The Compositional Appropriation of Instrumental Physicality to disrupt Pattern-based Musical Materials
}

\begin{abstract}
By Dr. Matthew Sergeant*
In recent years, many composers have explored a strategy to instrumental writing known as 'de-coupling'. In such work, the parameters involved in the physical operation of a musical instrument (e.g. slide position, tonguearticulation, etc.) are stratified into separate quasi-polyphonic musical layers - often notated on individual staves. The instrumentalist executes all of these parametric strands simultaneously, the moment-to-moment sonic result determined by the automatic reunification of the data-streams in performance (e.g. Aaron Cassidy's The Crutch of Memory (2004), for solo string instrument, or Klaus K. Hübler's Opus Breve (1988), for solo violoncello). A transposition of the above model has arisen as a result of my own research into this area in which the reunification of stratified parametric strands is prioritised as part of the compositional process; a

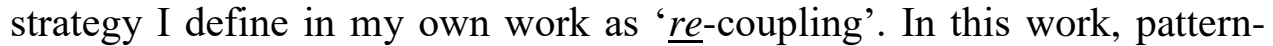
based musical materials (intervallic cycles, rhythmic loops, etc.) are composed independently for physically defined parametric strata of a single instrument (fingering, string-crossing, etc.) and then superimposed in the musical sketch. From here, only actions with significant sonic results are transcribed into the final score (instructions for fingers to depress unbowed strings, for example, are removed). The collision of the strata operates as a mutually affective filter, necessitating a disfiguration of the patterns they originally contained. More broadly, re-coupling allows hidden processes of elision to be reclaimed from a performative space and activated within a compositional methodology. An ecology of material is created where living musical behaviours compete to survive inside a shared instrumental habitat. This paper will demonstrate the applicability of these ideas to both micro and macro-scale musical design, with reference to recent examples from my own compositional work, and discuss the wider space created by this position: to what extent does the compositional and editorial act coalesce in this framework?
\end{abstract}

For some time now my compositional work has concerned what I have come to call activated notions of disruption as a methodology for the transformation of musical materials - that is to say, a compositional

\footnotetext{
*Royal Northern College of Music, Manchester, UK.
} 
grammatology where different, types, degrees or classes of disruption and distortion operate within the teleology of the same musical work. Such operation(s) can be considered as a plane in which to operate, the vectors of which are defined amongst my terminologies as erosion (removal) and encrustation (accrual). As part of the composing process, my materials are forced through this resultant space, their changing position resulting in differentiated states and levels of corruption, disintegration and ultimately transformation.

It is not the purpose of this paper to attempt to outline the widest holistic overview of my creative practice in these terms; what is attempted here is an outline of one of the many methodologies I have derived for composing in this activated/transformative space - an approach to instrumental writing that I have come to refer to as recoupling. The text that follows serves to locate the origin of this method within wider compositional approaches and demonstrate its application in two of my recent compositions: bet merkorios (2012), for solo violoncello, and ymrehanne krestos (2012-13), for flugelhorn, alto trombone and percussion. In doing so, it will be demonstrated how, and in what sense, this plane of erosion/encrustation explored via this technique.

The derivation of my recoupling strategy began with a reading of the various strategies employed under the term parametric decoupling, a broad label that generally encompasses a trend in recent decades towards certain strategic tropes in acoustic instrumental writing. As befits these kinds of generalised composition terms, it should obviously be acknowledged that the parametric decoupling label operates as an umbrella-term for a very broad compositional space, itself occupied by a number of composers, each with their own wildly divergent aesthetic and conceptual infrastructures (such as composers Aaron Cassidy, Frank Cox and Klaus K. Hübler and Claus-Steffan Mahnkopf, amongst many others). Whilst it is not the purpose of this paper to outline an annotated history of parametric decoupling as an emergent compositional strategy ${ }^{1}$, within the broad melange of practices that are encompassed by the term, three principal tropes emerge:

Physicality. In applicably decoupled musical work, the conceptual plane in which musical materials are constructed is wholly (if not in part) physically defined. That is to say that musical gestures are conceived in terms of, or indeed as the output of, the physical technical operations required to produce them on a given instrument. As Aaron Cassidy describes in relation to his composition for electric guitar The Pleats of Matter (2005-07):

The actions of the hands and arms are indicated in microscopic detail, and each physical motion - each hammered-on attack, each plucked string (above or below a fingered pitch), each glissando, each pitch-bend, each scrape of the string, each movement of the tremolo-bar - is carefully mapped out in such a way that the gestural action is itself already a musical object. Each action carries

${ }^{1}$ Simon Cummings provides an eloquent introduction to such historical precedents (Cummings 2012) 
a fundamentally musical set of data, and in fact much of the work revolves around a manipulation of the degree of dislocation between this physical movement and the actual resulting sound. (Cassidy 2008 pp.28-29)

In practice, this often results (especially within Cassidy's work) in the employment of various instrumental tablatures - notational strategies where information regarding the physical operation of the instrument is prescribed in minute detail, but the sonic result of these actions is not indicated.

Polyphony. With materials defined in physical terms in this way, a conceptual stratification of such materials can emerge as an operational framework. Consider the simple movement of a bow across a string (imagining any bowed string instrument will suffice for this thought experiment): the bow is drawn and a sound is produced. But the physical attributes of the motion here described are not as mono-dimensional as they first may appear; instead, it better considered as a composite action formed from several activated parameters. In actuality, any given bow stroke is operating in at least three parametric dimensions: (1) its left/right trajectory (up-bow or down-bow), (2) the pressure it exerts on the string and (3) its lateral position relative to the bridge. This process of physical disassembly can obviously be continued into several more dimensional planes, such as the speed of the bow across the string (4) or the angle through which it moves, which itself alters the string which is bowed (5a), or indeed the number of strings agitated in a single stroke (5b). Obviously, this thought processes can be transposed on to any physical instrumental action: consider the dimensional planes in operation in string instrumentalists' left hands, or those at play in keyed wind instruments.

The compositional value of such conceptual disassembly lies in the potential for independent treatment of these emerging dimensional spaces. The emerging model allows for separate musical strata to be constructed for each physical parameter and then superimposed. Distinct sets of gestural behaviours, for example, can now be constructed independently for (a) manipulation of lateral bow movement and (b) bow pressure.

In practice, much work of this nature is thus presented as multi-stave instrumental parts, each stave denoting separate physical parameters - and often making extensive use of polyrhythms to desynchronise the various strata.

In essence, a sense of parametric polyphony is created via the superimposition of such materials, although the extent to which the resultant polyphonic space occupies a constructional/compositional or perceptive/sonic realm is debated from composer to composer ${ }^{1}$.

Performance. Whether polyphony is a perfect conceptual term in this context or not, what remains clear is that the sonic surface of a work of this type is something of an assemblage; a hybrid formed from the superimposition - or perhaps collision is a better term - of initially notationally distinct parametric strata.

\footnotetext{
${ }^{1}$ Aaron Cassidy develops this discussion particularly eloquently (Cassidy 2002).
} 
But the physical origination of the material opens a wider space for consideration. The physicality of these works' gestural layers allow for probably their best understanding in physical terms, as forces. As two or more directional forces collide in nature, a hybrid output force is formed by the collision. The attributes of this output force (its velocity, trajectory, etc.) are wholly dependent on the initial inputs that created it; some combinations will cancel each other out, others will amplify one another. Aaron Cassidy describes musical manifestations of such phenomena in relation to his composition metallic dust (1999) for solo bass clarinet, which collides the parametrically segregated strands of the mouth and fingers of the instrumentalist:

The lower staff is used to designate the action of the fingers in depressing the keys (and the resulting pitches) and is notated in the typical fashion. However, it must be noted that certain physical actions indicated here on this second staff will not be readily audible: when finger action is called for without corresponding mouth action, the player is instructed to adhere to the "fingers" information with utmost sobriety. These silent passages are not intended to be at all parodistic, nor are they to be exaggerated in any way, but are simply performed in their typical manner [...]. It should be understood that the unpredictability of such "de-coupled" interaction is the composer's intentions; unstable transitional and inexact sounding results will emerge from the interaction of the two strands with a rhythmic profile which extends far beyond even the already-precise notation on the page. (Cassidy 2002 pp.151)

As such, the collision of, now musical, forces can be considered as a transformative operation in action upon the score: the output result (the sonic surface) being simultaneously both and neither of its constituent input strands.

From the perspective offered by the particular work described above, such transformation can also be considered as the result of a destructive force: in the collisions of various physical strata, Cassidy describes the expectation for omissions ('silences') from the original score data and rhythmic results wildly divergent from that indicated on paper. The destructive nature of these forces has not gone unnoticed by commentators, as Evan Johnson demonstrates:

The earliest works, the wind solos metallic dust (1999) and asphyxia (2000), are contrapuntal dances between pitches and the forces that distort them, melodic shapes and cantus firmi are damaged by independently manipulated embouchure and breath before they escape the instrument. (Johnson 2012 pp.5)

But in what realm do these damaging operations occur?

It has already been outlined how the disruptive forces generated within the music are the result of the collision of parametric strata. But these collisional 
forces only become activated when the work is executed in performance. From a certain conceptual angle then, in this music, the destructive transformative plane is only operational in the performative realm and actually lies dormant in the score itself. That is to say that there is little-to-nothing intrinsically disrupted or distorted in the raw constituent material as printed on the page, such transformative properties are only accessed when a given work is realised through performative execution in the concert hall.

Such reasoning led me to my own speculation: how could the destructive forces instigated by particular applications of so-called parametric decoupling be reclaimed from this performative space and reintroduced into the realm of the score-object itself? In other words, how could the fundamental tenets and principals hitherto outlined be re-appropriated to instigate a scenario where similar collisional and explosive forces are operational in a compositional realm, resulting in material on the page that can be considered as already the result of distortion or disruption, prior to its enacting via the music stand.

My first composition to explore this line of enquiry is entitled bet merkorios (2012), for solo violoncello. In essence, the piece can be considered as a large-scale study of the compositional re-appropriation in the forces of omission, as documented in relation to Cassidy's metallic dust.

The construction of the first three bars of my composition (figure 1) is equitable to the strategy employed throughout the composition as a whole: the score-surface - that is to say the music printed on the finished page - is the result of a controlled collision of materials in compositional space.

Figure 1. Matthew Sergeant; bet merkorios (2012) for solo violoncello; bars 1-3

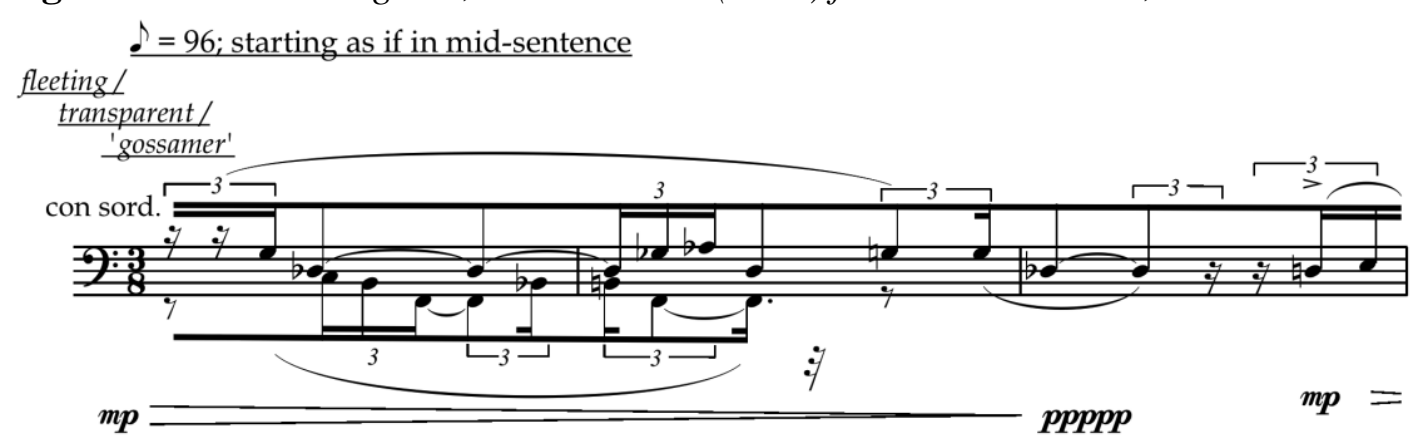

(In reading the examples provided, it will also be necessary for the reader to consider that the composition employs a fairly divergent scordatura, illustrated in figure 2).

Figure 2. Matthew Sergeant; bet merkorios (2012) for Solo Violoncello; Scordatura 


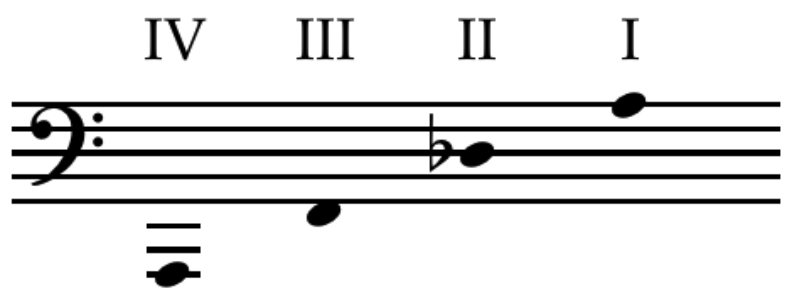

Reminiscent of the 'polyphonic' attributes of parametrically decoupled compositional work, the substrata from which the score-surface of bet merkorios is constructed consists of two simultaneously operational streams of parametric data, each controlling one of the cellists' hands (left-hand, fingerboard; right-hand, bow). In each parametric stratum, the material can be considered as pattern ${ }^{l}$ based. That is to say that, taken in a form abstracted from the completed score-surface, the behaviour of each parametric thread produces highly consistent musical data (thus creating a consistency which may be disrupted). Eleven different patterns are utilised in the composition; five define fingerboard behavior (i.e. the activity of the left hand) and six define the behavior of the bow (i.e. the activity of the right hand). By permutating which pair of patterns are operational at any one time, a variety of scenarios are produced, i.e. a given left hand pattern may be collided with several different right hand comportments throughout the course of the composition, and vice versa.

During the opening bars, the first left hand (fingerboard) comportment utilized is that of a cyclic fingering pattern. Part of the definitive behavior - or comportment, to use the terminology of my own compositional sketches - of this pattern's textural trope involves the combination of consistent gestural behavior both across the strings (from IV-I) and 'up' and 'down' the fingerboard.

In order to control such bidirectional movement, the fingerboard of instrument was delineated into eight fixed, non-overlapping, hand positions, each hand position allowing physical access to four possible pitches (via the four fingers of the left hand). This delineation can be visualized as figure 3 .

In this tabulation (figure 3), the circled Arabic numerals on the left indicate the eight designated hand positions. The lowest stave shows the string to which each a hand position is applied, enumerated with the traditional Roman numerals from IV (lowest) to I (highest). The space enclosed by this table was then used to construct a pitch-cycle, designed to allow physically seamless executable repetitions (i.e. the hand is in a suitable position at the end of the cycle to immediately facilitate an additional iteration). The resultant cycle was then transposed through all available hand positions. This process

\footnotetext{
${ }^{1}$ Ultimately, the employment of the term pattern is a reference to Feldman's compositional practice (Feldman 2001 pp.134-149) but perhaps the term can better understood in relation to Elliott Carter's employment of 'character-patterns', where 'intervals, metronomic speeds, polyrhythms and rhythmic characters [are] used to dramatize the musical personalities of textures' (Schiff 1998 pp.36)
} 
can be visualized as figure 4 (the lowest staff, designating the open strings on which all the notes in the column above is performed, is only provided for information-only: open strings are never outputted as part of this pattern). Controlled movement through this table (figure 4) created the first left-hand parametric data-stream used in the composition. 
Figure 3. Hand Positions and Fingerings as defined within Bet Merkorios

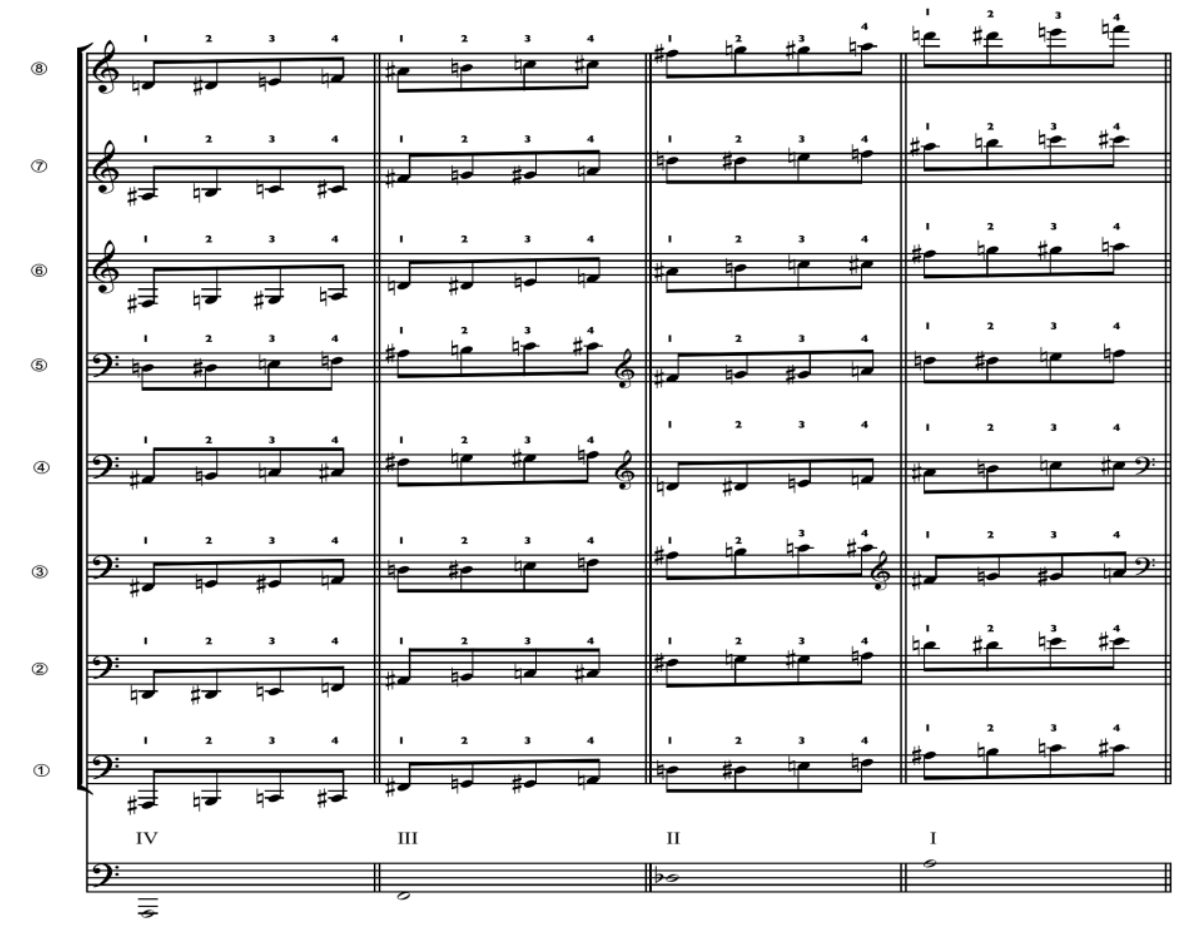

Figure 4. Left-hand Cyclic Material employed in in Bet Merkorios (b.1-3)

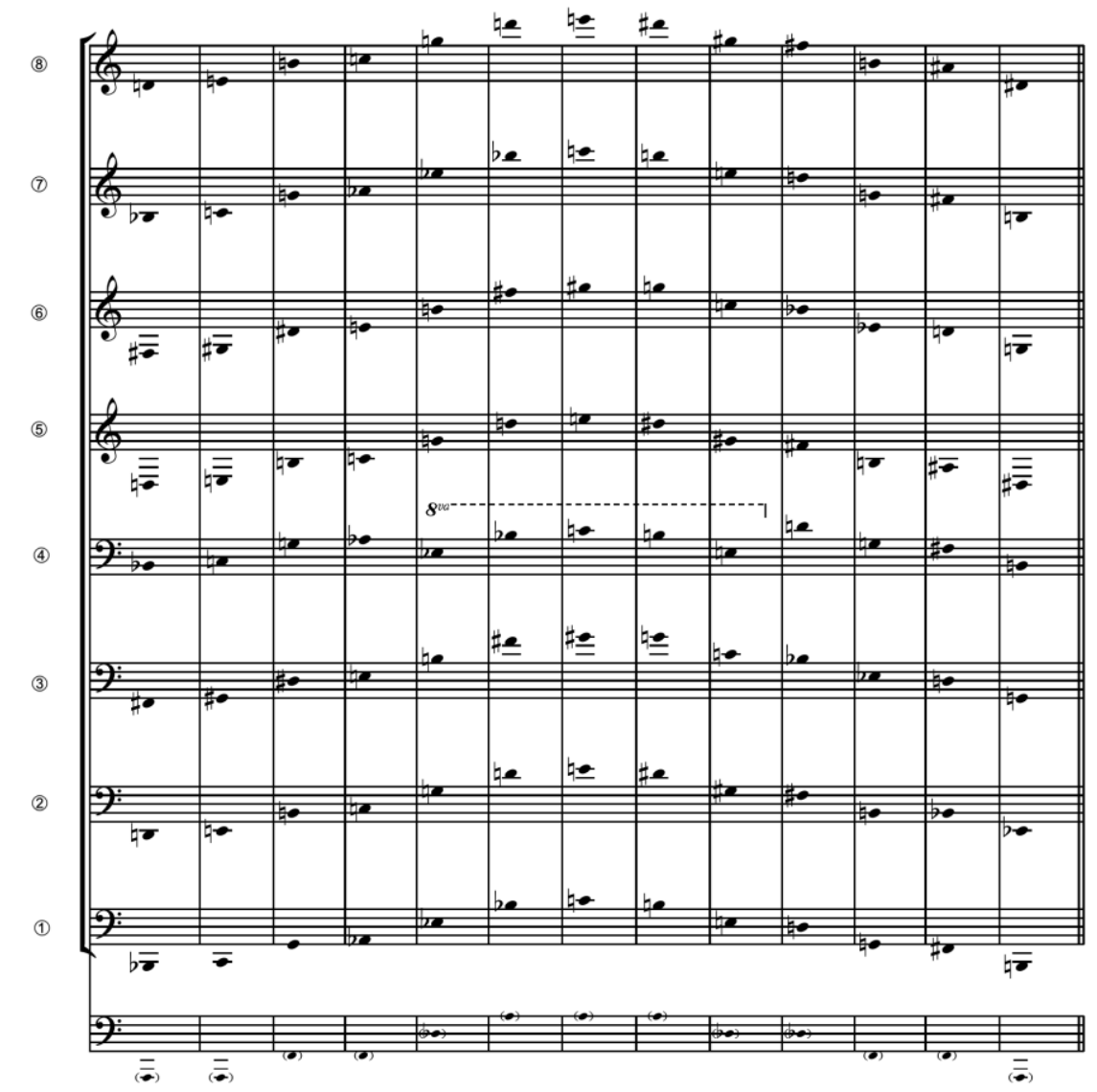


Although it is beyond the scope of this paper to detail its mechanisms here, it is worth outlining that such movement was generated in computer software and was statistically controlled to maintain internal consistency. The output of the statistical framework used includes (relative to motion within the table), sporadic changes of direction within the cycle and occasional vertical movements to a new hand position (the frequency and size of leaps again being chosen in relation to an additional probability distribution). Rhythmically, the data output from this particular pattern was characterized as a perpetuum mobile (using only triplet-semiquaver values) and can be seen in figure 6 .

The consistency detailed in relation to this pattern is corrupted via its collision with an additional pattern, now conceived of entirely within the operational parameters of right (bow) hand.

At the opening of the composition, the operational right-hand pattern can be described as a rhythmicised random walk through a cycle of single and double-stopped open strings (again, produced via statistical control, for consistency). The pitch cycle (defined by the open retuned strings) within which the pattern operates can be seen in figure 5 .

Figure 5. Abstraction of Right-hand Cyclic Material, employed in in Bet Merkorios (b.1-3)

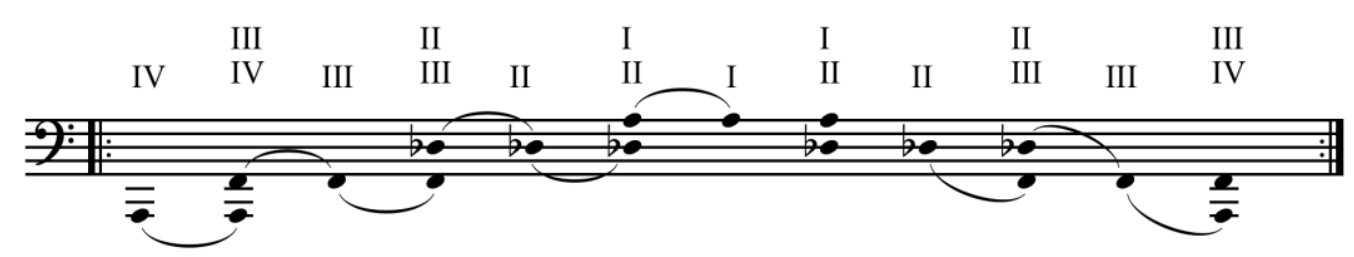

These parametric strata can now be superimposed in compositional space, a process that can be visualised as figure 6 .

Figure 6. Collision of Left and Right-hand Patterns in the Formation of Bet Merkorios, Bars 1-3. (Some performance instructions have been removed for ease of reading)

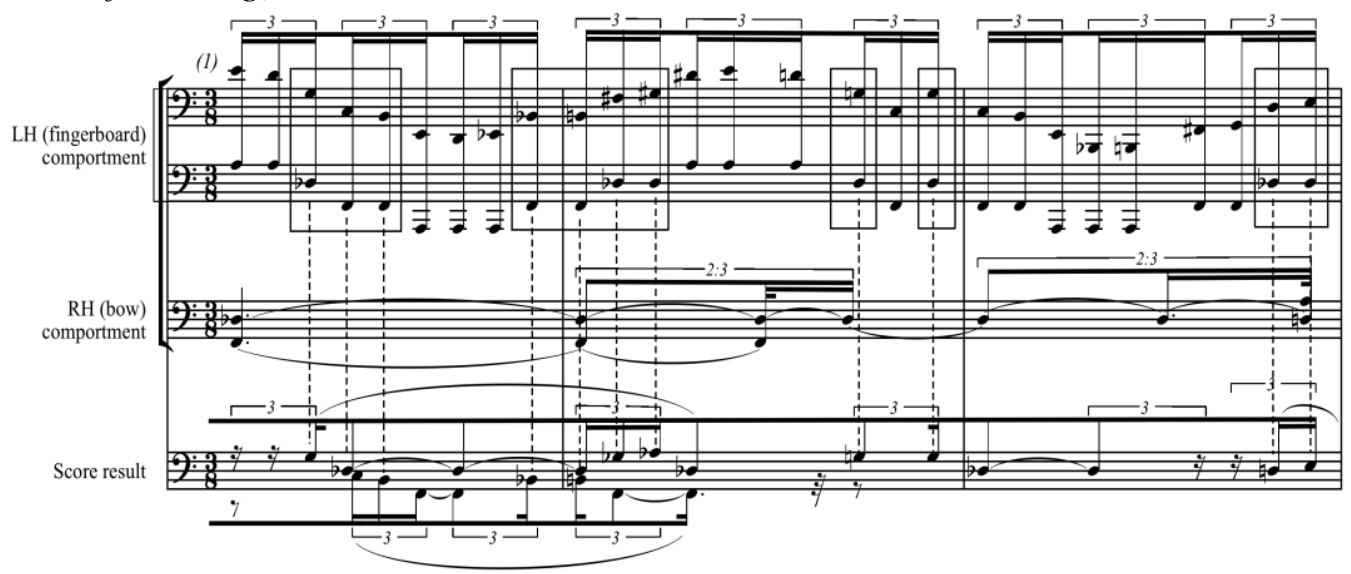


In effect, the two strata are filtered through one another, as if in performance, and only results with sonic consequence pass into the final scoresurface. Throughout bar 1, by way of example, only strings II and III are being stimulated by the bow (as determined by the bow's patterning logic), thus finger activity on any other strings (I and IV) induces 'null' results (silence). Such results are removed as the material passes to the final score-surface.

This collisional process can certainly be understood as a violence towards the original material. By omitting notes from material originally designed to operate as an internally consistent pattern, extraneous data is allowed to gather in the score-surface. Intervals not induced by the original pitch cycle are instigated and gradients and contours implied by the movement of the bow through pitch-space are undermined.

Obviously, the surface result of this process is intrinsically dependent on the permutation of the different parametric materials superimposed. Each particular pairing of materials occupies a wildly different erosional state some exposit a preference for preservation of material of the left hand, others the right. The qualitative difference between such pairings is exploited as part of the macrostructural strategy of bet merkorios; patterns are alternated, replaced and developed within their relevant physical planes throughout the composition's larger durational span.

The collisional process itself can be further understood as a conceptual relocation of instrumental physicality into the compositional process. As such, the conceptual framework around the distortive forces at play here is also transposed. In both my own work and the parametrically decoupled music hitherto discussed there are notions of object, impact and debris. In Cassidy's work, notational objects are impacted by the destructive force of performative action, producing sonic debris. In my own strategy, the same force is applied to abstracted physical objects, producing notational debris. In Cassidy, the destructive operation takes place within the temporality of the sonic work in performance, in my own, the impact is external, rendering its operative forces invisible.

The imagined image of debris can be confused with that of ruin something that was once whole, now incomplete - but the byproduct of colliding destructive forces may not be so. Forces can also transform through amplification, as if two motions along the same vector conjoin. To return to the imagination, the debris is now an additive surface on the whole, like barnacles changing the outline of a submerged vessel, or lichens and mosses on a stone.

In light of such thought, the destructive powers at work in bet merkorios occupy a single conceptual directionality; that of erosion and omission as transformative operands. What if this movement through compositional space was reversed, the forces at work now serving to encrust and engulf, rather than remove and corrode?

The opportunity to move in this opposite vector - one of encrustation rather than erosion - came via my composition ymrehanne krestos (2012-13), for flugelhorn, alto trombone and percussion. Again, in this second composition, the conceptual planes from which the material was harvested are 
physically conceived, particularly in relation to construction of the music for the brass. In selecting the parametric dimensions in which to operate, care was taken to move along parallel planes that could thus, in essence, coexist within the same musical moment without omission. The destructive forces sought are thus invoked by the mutual encrustation of these layers as they entwine in the score-surface.

To this end, the brass music of ymrehanne krestos is the result of four entangled parametric layers: (1) articulation, the physical action of the tongue inside the mouth; (2) valve combination (or, indeed, slide position); (3) airpressure, essentially the harmonic partial activated by the air-column inside the instrument at any one time; (4) air-speed, 'dynamics'. Four material patterns were then independently designed for each of the four resulting parametric spaces, in a manner akin to those employed within bet merkorios. All are independently rhythmicised; the same four parametric layers are employed in both the flugelhorn and trombone.

As such, the opening bar of flugelhorn material from the composition can be tabulated in relation to its constituent parametric strata (figure 7).

Figure 7. Matthew Sergeant: Ymrehanne Krestos; Flugelhorn (transposed); b.1; Score-result with Constituent Parametric Stratal

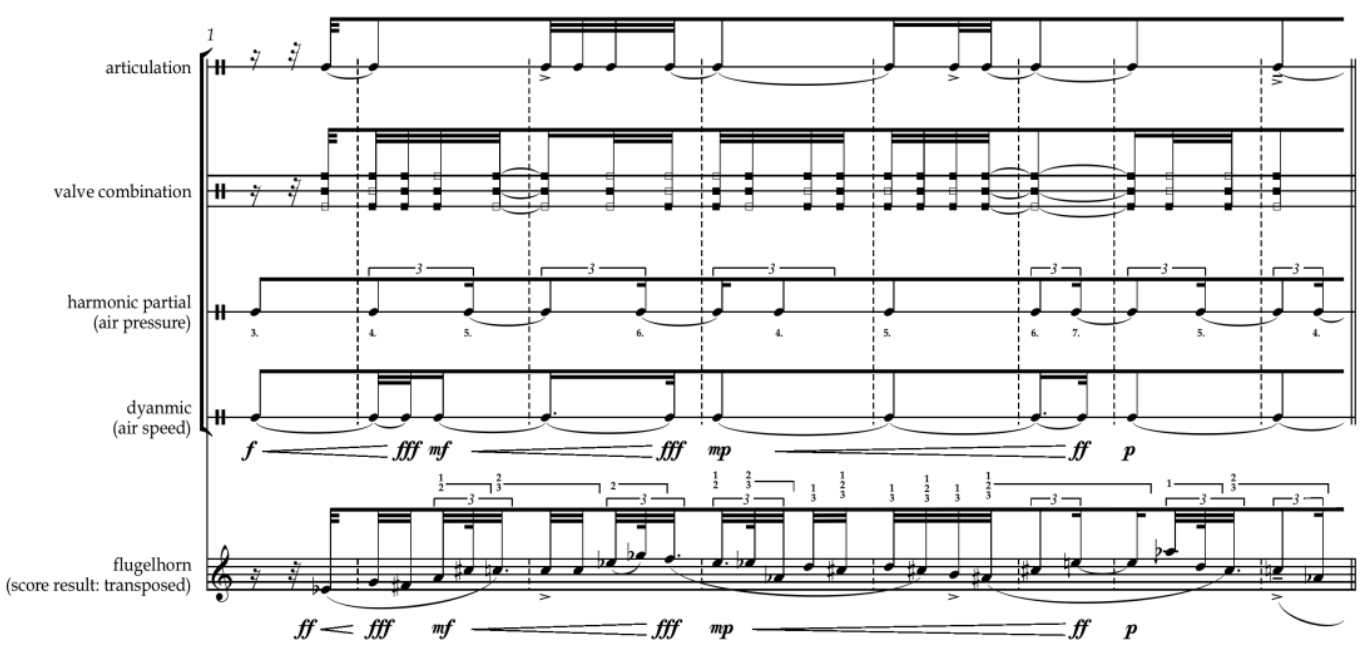

Whilst the above example illustrates the distortive power of localized parametric encrustation (the contours of the harmonic partials are encrusted -

\footnotetext{
${ }^{1}$ In case further clarification is needed, from top to bottom the staves indicate (1) articulation; (2) valve combination, the three-line stave indicating the three valves of the instrument as seen from the perspective of the player (lowest line, valve-1; middle-line, valve-2; top-line, valve$3)$; (3) harmonic partial to be played (regardless of the fundamental instigated by the valvecombination); (4) dynamics; (5) score result - the outcome if all the top set of materials were to be performed simultaneously. Only the score result is presented to the player in the finished version. Dotted vertical bar lines are provided here to delineate beats, for reasons of clarity only. Where the superimposition results in atypical valve-combinations for certain pitches (e.g. $2+3$ for notated C5) the valve combination to be used is explicitly stated in the score result and the performers' parts
} 
and thus eroded - by the localized pitch contours of the valve mechanism; the dynamic gestures serve only to undermine the phrase-structure outlined by the tongue; etc.), an additional level of interference is obtained with regard to the relationship between the brass instruments:

At a slightly deeper compositional level, all four parametric patterns share a hocketing behavioral trope - that is to say all four parametric patterns (in their abstracted state prior to combination in the score surface) alternate material across the two brass instruments. The eight parametric strata in operation in bar 1 (four in the flugelhorn, four in the trombone) can thus be reconsidered as four simultaneous parametric duos (figure 8).

Figure 8. Matthew Sergeant: Ymrehanne Krestos; Flugelhorn and Alto Trombone; b.1; Four Simultaneous Parametric Duos

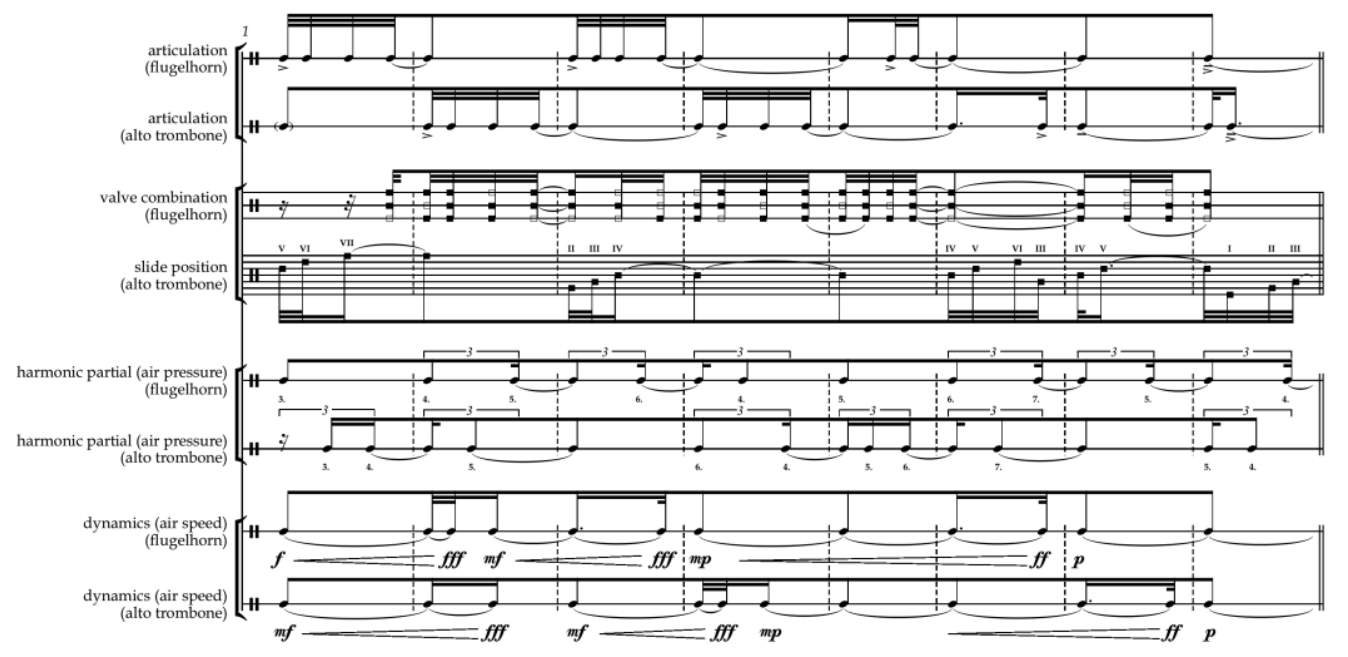

The destructive power of encrustation is once again best considered here in terms of force. In any given hocket, parametric activity is constantly oscillating between instruments. A vectorial force is essentially created, from instrument to instrument and back again. At any given moment (particular in this and the immediately surrounding bars), four such vectorial forces are thus in operation (four hockets; four forces). The oscillation of the hockets is not temporally synchronized, leading to the resulting gestural forces between instruments 'crossing paths'. In itself, this leads to a diverse array of scenarios. The motions of oscillating parametric activity across the ensemble can be effectively cancelled out; as certain parameters momentarily stabilize, others become active again, at no point is a sense of holistic stasis ever felt. In contrast, the oscillations can amplify one another, as all four hockets momentarily and arbitrarily align. In either case - and particular the grey areas between the two - the instrumental behavior of the ensemble is in a constant state of destabilization and flux; no hocket or pattern is allowed to 'speak' with any purity at any compositional level.

This re-appropriation of instrumental physicality into the compositional domain - this recoupling - can thus be viewed from the perspective of its own tripartite set of conceptual tropes. 
Recoupling can be read as a relocation of the destructive potential displayed in decoupled instrumental work into the compositional plane: whereas the operative plane in the former is performative, recoupled work is compositive (to make composite). In turn, the composite nature of the work no longer plays comfortably with polyphony. In both of my compositions here discussed, polyphony is a pre-existent state, the resultant score surface is singular/ composite/residue; it is already only a remnant of past collision. In a sense, this journey from pre-existence to remnant is irreversible. The score belies only traces of the forces that created it: the polyphony can never be fully resurrected from its result (in the mind of the performer, or even analyst). Such irreversible powers are those of fusion: material species are forced into deformed hybrids, simultaneously both and neither of the ancestral forms from which they are comprised. As Deleuze says

The transformation of form can be abstract or dynamic. But deformation is always bodily, and it is static, it happens at one place; it subordinates movement to force but it also subordinates the abstract to the Figure. When a force is exerted on a scrubbed part, it does not give birth to an abstract form, nor does it combine sensible forms dynamically: on the contrary, it turns this zone into a zone of indiscernibility that is common to several forms, irreducible to any of them [...] (Deleuze 2003 pp.42)

My material emerges from the body but is disfigured before it becomes sonic/heard. The nature of the material - the physicality of the material - is depurified as it collides with itself into the score surface. The composite produced is no longer entirely of the body, it has solidified into a singular notational object.

In the concert hall, the performative execution of the score serves now not to resurrect the bodily origins of the material, but as a vehicle for the contorting forces that created it. Through this performative vehicle, the remnant releases haptic reverberations of the violence of its construction - the compositions' sonic surface. The source of these reverberations are felt within the nature of the sonic-ised material: in emerging into sound alongside its formative violence, what was a notational object now exists audibly only as debris.

\section{Bibliography}

Cassidy, Aaron. "Determinate Action/Indeterminate Sound: Tablature and Chance in Several Recent Works." In Facets of the Second Modernity. Hofheim: Wolke Verlag, 2008.

Cassidy, Aaron. "Interconnectivity and Abstraction: Metallic Dust As a Testing Ground for Monophonic and Strucutral Polyphonies." In Polyphony \& Complexity. Hofheim: Wolke Verlag, 2002.

Cummings, Simon. Giving voice to the indescribable: Aaron Cassidy - The Crutch of Memory. Accessed 21 April 2013, http://5against4.com/2012/05/28/giving-voice- 
Vol. 1, No. 3 Sergeant: Introducing 'Re-coupling'...

to-the-indescribable-aaron-cassidy-the-crutch-of-memory/, 2012

Deleuze, Gilles. Francis Bacon: The Logic of Sensation. Translated by Daniel W Smith. New York; London: Continuum, 2003.

Feldman. "Crippled Symmetry." In Give My Regards to Eighth Street: Collected Writings of Morton Feldman. Boston, Mass.: Exact Change; Turnaround, 2001.

Johnson, Evan. Works by Aaron Cassidy (CD Liner Notes). München: NEOS (Recording No.: NEOS 11201), 2012.

Schiff, David. The Music of Elliott Carter. London: Faber, 1998. 
